\title{
Rivulet flow over and through a permeable membrane
}

\author{
Abdulwahed S. Alshaikhi $\odot,{ }^{*}$ Stephen K. Wilson $\odot,^{\dagger}$ and Brian R. Duffy $\oplus^{*}$ \\ Department of Mathematics and Statistics, University of Strathclyde, Livingstone Tower, \\ 26 Richmond Street, Glasgow G1 1XH, United Kingdom
}

(Received 26 May 2021; accepted 13 September 2021; published 7 October 2021)

\begin{abstract}
Motivated by small-scale natural and industrial processes involving flow over and/or through a layer of a porous medium, a mathematical model for the steady gravity-driven flow of a rivulet of fluid with finite width over and through a permeable membrane is formulated and analyzed. The three-dimensional shape of the free surface of a rivulet with either fixed semiwidth or fixed contact angle is determined, and it is shown how the length, base area, and volume of the rivulet on the permeable part of the membrane depend on the physical properties of the system. In particular, whereas there is a physically realizable pendant rivulet solution only if the semiwidth does not exceed a critical value, there are physically realizable sessile and vertical rivulet solutions for all values of the semiwidth; moreover, a sessile rivulet with fixed semiwidth has a finite maximum possible length which is attained in the limit of a wide rivulet.
\end{abstract}

DOI: 10.1103/PhysRevFluids.6.104003

\section{INTRODUCTION}

There are many natural and industrial situations in which a layer of fluid flows over and through a layer of a porous medium. While there is a substantial body of theoretical and experimental work motivated by large-scale flows, such as tidal motion over a sandy beach, the possible escape of liquidized carbon dioxide sequestered in porous underground reservoirs, and the flow of lava over fractured bedrock, including that by Ungarish and Huppert [1], Acton et al. [2], Pritchard et al. [3], Sayag and Neufeld [4], Liu et al. [5], and Yu et al. [6], the motivation for the present work is the small-scale flows that occur in processes such as the absorption of pesticides by the leaves of plants (see, e.g., Tredenick et al. [7]), coating and printing onto porous substrates (see, e.g., Clarke et al. [8]), dyeing textiles (see, e.g., Shamey and Zhao [9]), the flow of condensed water on mesh fog-harvesting devices (see, e.g., Park et al. [10]), and screen printing (see, e.g., White et al. [11]), all of which involve flow over and/or through porous layers. In order to enhance our understanding of such situations, in the present work we analyze the steady gravity-driven flow of a rivulet (or trickle) of fluid over and through a permeable membrane. In particular, we seek to determine how far the rivulet flows over the membrane before it is completely imbibed by it.

\footnotetext{
*abdulwahed.alshaikhi@strath.ac.uk

${ }^{\dagger}$ Author to whom correspondence should be addressed: s.k.wilson@strath.ac.uk.

b.r.duffy@strath.ac.uk
}

Published by the American Physical Society under the terms of the Creative Commons Attribution 4.0 International license. Further distribution of this work must maintain attribution to the author(s) and the published article's title, journal citation, and DOI. 
A landmark contribution to the understanding of small-scale flow over and through a porous layer is the work by Davis and Hocking [12,13] on the spreading and imbibition of a droplet of fluid on a porous substrate, which has been extended and generalized by many subsequent authors, notably Clarke et al. [8], Starov et al. [14,15], Allerborn and Raszillier [16], Anderson [17], Nong and Anderson [18], and Espín and Kumar [19,20]. Even more directly relevant to the present study is the analysis of Davis and Hocking [12] of the steady flow of a two-dimensional fluid sheet over and through a porous substrate and, in particular, their calculation of the length of the sheet (referred to by them as its "survival distance") on the permeable part of the membrane. They did not, however, consider three-dimensional situations such as the present rivulet flow. Also relevant here is the work by Liu and Liu [21], Kandel and Pascal [22], and Iervolino et al. [23] on the stability of the flow of a two-dimensional fluid sheet down an inclined porous layer, and that by Ramon et al. [24], Knox et al. [25,26], and Venerus [27] on porous squeeze-film flow, all of which take account of the flow of fluid into and/or out of the porous layer. The review by Gambaryan-Roisman [28] provides a helpful overview of work on the wetting of porous layers.

Many aspects of rivulet flow over impermeable substrates have been studied by previous authors. In the absence of a recent review, the works by, e.g., Paterson et al. [29], Alekseenko et al. [30], Herrada et al. [31], Howell et al. [32], Al Mukahal et al. [33], Wang et al. [34], Alshaikhi et al. [35], Ledda et al. [36], Aktershev et al. [37], and the references therein, provide a representative selection of these previous studies.

\section{MATHEMATICAL MODEL}

\section{A. Problem formulation}

Consider the steady gravity-driven flow of a symmetric rivulet of an incompressible Newtonian fluid with constant density $\rho^{*}$, viscosity $\mu^{*}$, and surface tension $\sigma^{*}$ over and through a planar membrane of constant thickness $H^{*}$ inclined at an angle $\alpha(0 \leqslant \alpha \leqslant \pi)$ to the horizontal. In particular, sessile $(0 \leqslant \alpha<\pi / 2)$, vertical $(\alpha=\pi / 2)$, and pendant $(\pi / 2<\alpha \leqslant \pi)$ rivulets are all considered. As shown in Fig. 1, the membrane is impermeable for $x^{*} \leqslant 0$, but permeable with uniform permeability $k^{*}$ for $x^{*}>0$. To describe this situation we use Cartesian coordinates $O x^{*} y^{*} z^{*}$ with the $x^{*}$ axis down the line of greatest slope, the $y^{*}$ axis horizontal, and the $z^{*}$ axis normal to the "top" surface of the membrane (i.e., the surface of the membrane with the rivulet on it) $z^{*}=0$. The passive atmosphere surrounding (i.e., both above and below) the rivulet and the membrane is assumed to be at constant atmospheric pressure $p_{\mathrm{a}}^{*}$. On the impermeable part of the membrane, $x^{*} \leqslant 0$, the rivulet flows over the membrane, the flow within it is strictly unidirectional in the $x^{*}$ direction, and it has uniform free surface profile $z^{*}=h^{*}\left(y^{*}\right)$, constant volume flux in the positive $x$ direction $\bar{Q}^{*}(>0)$, constant semiwidth $\bar{a}^{*}(>0)$, and constant contact angle $\bar{\beta}^{*}(>0)$. (As we shall show subsequently, the values of $\bar{Q}^{*}, \bar{a}^{*}$, and $\bar{\beta}^{*}$ are related to each other, and so only two of them may be prescribed independently.) On the other hand, on the permeable part of the membrane, $x^{*}>0$, the rivulet again flows over the membrane but now fluid also flows out of the base of the rivulet and through the membrane, causing the rivulet to shrink, and so the flow within it is no longer strictly unidirectional, and it has nonuniform free surface profile $z^{*}=h^{*}\left(x^{*}, y^{*}\right)$, nonconstant volume flux in the positive $x$ direction $Q^{*}\left(x^{*}\right)\left(0 \leqslant Q^{*} \leqslant \bar{Q}^{*}\right)$, nonconstant semiwidth $a^{*}\left(x^{*}\right)\left(0 \leqslant a^{*} \leqslant \bar{a}^{*}\right)$, nonconstant contact angle $\beta^{*}\left(x^{*}\right)\left(0 \leqslant \beta^{*} \leqslant \bar{\beta}^{*}\right)$, and length $L^{*}(>0)$. The rivulet is continuous as it passes from the impermeable to the permeable part of the membrane, and so, in particular, $Q^{*}, a^{*}$, and $\beta^{*}$ take the "initial" values $\bar{Q}^{*}, \bar{a}^{*}$, and $\bar{\beta}^{*}$, respectively, at $x^{*}=0$. Eventually (i.e., sufficiently far downstream from $x^{*}=0$ ) the rivulet is completely imbibed by the membrane (i.e., $Q^{*}$ becomes zero). The determination of how the length of the rivulet on the permeable part of the membrane, $L^{*}$, depends on the physical properties of the system is one of the key aspects of the present work. We assume that once the fluid from the rivulet has passed through the membrane it plays no further role in the problem, i.e., there is no fluid on the "bottom" surface of the membrane (i.e., the surface of the membrane without the rivulet on it) 


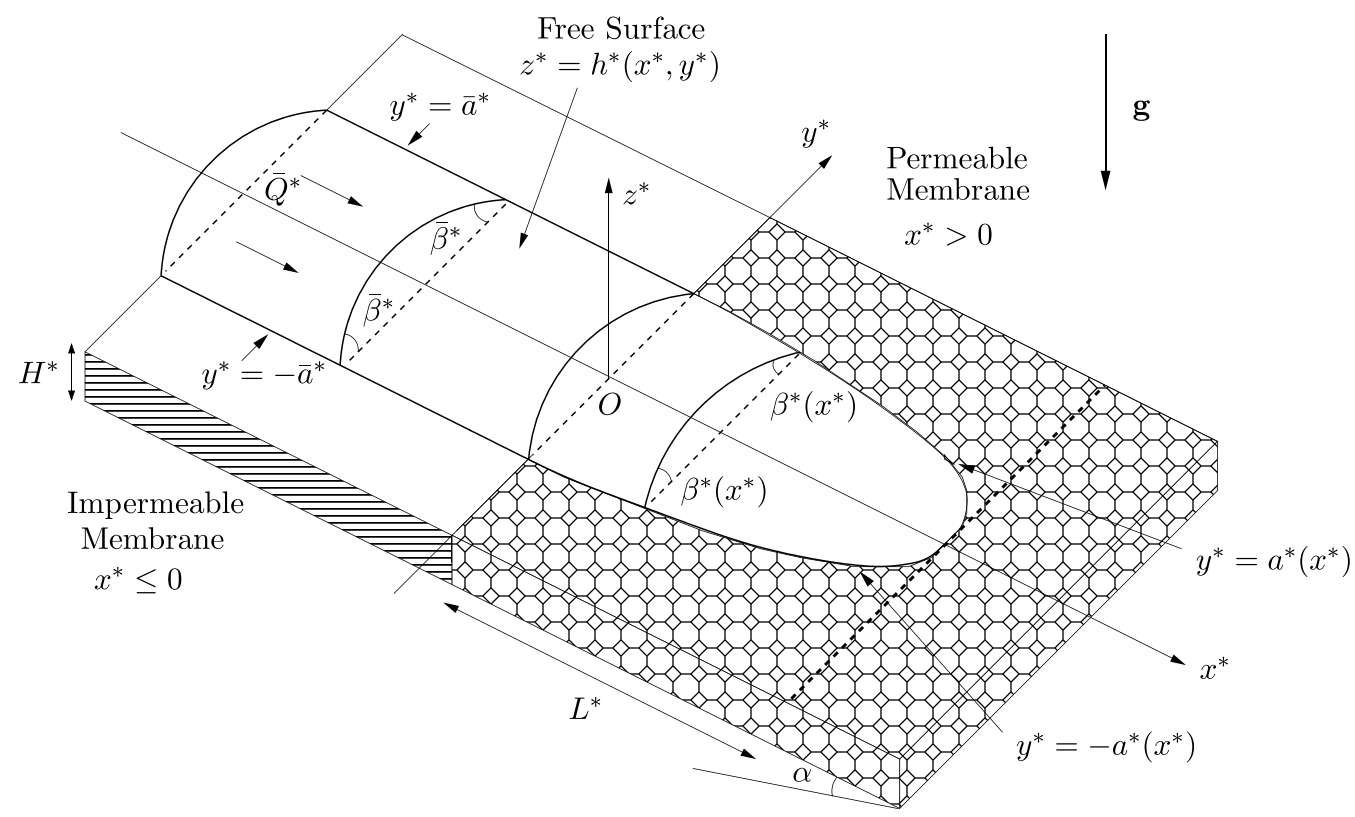

FIG. 1. Geometry of the steady gravity-driven flow of a rivulet over and through a planar membrane of constant thickness $H^{*}$ which is impermeable for $x^{*} \leqslant 0$, but permeable with permeability $k^{*}$ for $x^{*}>0$, inclined at an angle $\alpha(0 \leqslant \alpha \leqslant \pi)$ to the horizontal.

$z^{*}=-H^{*}$ [38]. In the present work we will consider both the case of a rivulet with constant semiwidth $a^{*} \equiv \bar{a}^{*}$ but decreasing contact angle $\beta^{*}\left(x^{*}\right)\left(\leqslant \bar{\beta}^{*}\right)$ in $x^{*}>0$ in Sec. III, and the case of a rivulet with constant contact angle $\beta^{*} \equiv \bar{\beta}^{*}$ but decreasing semiwidth $a^{*}\left(x^{*}\right)\left(\leqslant \bar{a}^{*}\right)$ in $x^{*}>0$ in Sec. IV.

Within the rivulet, the velocity $\boldsymbol{u}^{*}=\left(u^{*}\left(x^{*}, y^{*}, z^{*}\right), v^{*}\left(x^{*}, y^{*}, z^{*}\right), w^{*}\left(x^{*}, y^{*}, z^{*}\right)\right)$ and the pressure $p^{*}=p^{*}\left(x^{*}, y^{*}, z^{*}\right)$ satisfy the mass conservation equation, $\nabla^{*} \cdot \boldsymbol{u}^{*}=0$, and the Navier-Stokes equation. On the other hand, within the permeable part of the membrane, the Darcy velocity (i.e., the volume flux of fluid per unit area) $\boldsymbol{U}^{*}=\left(U^{*}\left(x^{*}, y^{*}, z^{*}\right), V^{*}\left(x^{*}, y^{*}, z^{*}\right), W^{*}\left(x^{*}, y^{*}, z^{*}\right)\right)$ and the pressure $P^{*}=P^{*}\left(x^{*}, y^{*}, z^{*}\right)$ satisfy the mass conservation equation, $\nabla^{*} \cdot \boldsymbol{U}^{*}=0$, and Darcy's law, $\boldsymbol{U}^{*}=-\left(k^{*} / \mu^{*}\right)\left[\nabla^{*} P^{*}-\rho^{*} \boldsymbol{g}^{*}\right]$, where $\boldsymbol{g}^{*}$ denotes acceleration due to gravity, and hence $P^{*}$ satisfies Laplace's equation, $\nabla^{* 2} P^{*}=0$. On the free surface of the rivulet, $z^{*}=h^{*}$, the usual continuity of normal and tangential stress and kinematic conditions apply. On the top surface of the membrane, $z^{*}=0$, we impose no flow in the tangential directions (i.e., in the $x^{*}$ and $y^{*}$ directions) [39] together with no flow in the normal direction (i.e., in the $z^{*}$ direction) on the impermeable part of the membrane and continuity of both pressure and volume flux in the normal direction on the permeable part of the membrane. On the bottom surface of the membrane, $z^{*}=-H^{*}$, we impose continuity of pressure.

\section{B. Scaling and nondimensionalization}

In the present work we consider the case of a slowly varying (in the $x$ direction) and thin (in the $z$ direction) rivulet flowing over and through an even thinner (in the $z$ direction) membrane, specifically the situation in which the longitudinal aspect ratio of the rivulet $\epsilon \ll 1$, the transverse aspect ratio of the rivulet $\delta_{\mathrm{R}} \ll 1$, and the transverse aspect ratio of the membrane $\delta_{\mathrm{M}} \ll 1$ are all small, and satisfy $\epsilon \ll \delta_{\mathrm{R}}$ and $\delta_{\mathrm{M}} \ll \delta_{\mathrm{R}}$. We anticipate that the closely related situation in which the rivulet and the membrane are thin but the thickness of the membrane is comparable to that of 
the rivulet, i.e., when $\delta_{\mathrm{M}} \sim \delta_{\mathrm{R}} \ll 1$, in which gravitational effects will play a more significant role within the membrane, could be analyzed using the same approach as that described in the present work.

To analyze this situation we scale and nondimensionalize the variables describing the flow of the rivulet according to

$$
\begin{aligned}
& x^{*}=\frac{\ell^{*}}{\epsilon} x, \quad L^{*}=\frac{\ell^{*}}{\epsilon} L, \quad y^{*}=\ell^{*} y, \quad a^{*}=\ell^{*} a, \quad z^{*}=\delta_{\mathrm{R}} \ell^{*} z, \quad h^{*}=\delta_{\mathrm{R}} \ell^{*} h, \quad \beta^{*}=\delta_{\mathrm{R}} \beta, \\
& u^{*}=\frac{\delta_{\mathrm{R}}^{2} \rho^{*} g^{*} \ell^{* 2}}{\mu^{*}} u, \quad v^{*}=\frac{\epsilon \delta_{\mathrm{R}}^{2} \rho^{*} g^{*} \ell^{* 2}}{\mu^{*}} v, \quad w^{*}=\frac{\epsilon \delta_{\mathrm{R}}^{3} \rho^{*} g^{*} \ell^{* 2}}{\mu^{*}} w, \quad Q^{*}=\frac{\delta_{\mathrm{R}}^{3} \rho^{*} g^{*} \ell^{* 4}}{\mu^{*}} Q, \\
& p^{*}=p_{\mathrm{a}}^{*}+\delta_{\mathrm{R}} \rho^{*} g^{*} \ell^{*} p,
\end{aligned}
$$

and the additional variables describing the flow in the membrane according to

$$
\begin{gathered}
z^{*}=\delta_{\mathrm{M}} \ell^{*} Z, \quad H^{*}=\delta_{\mathrm{M}} \ell^{*} H, \quad k^{*}=\epsilon \delta_{\mathrm{R}}^{2} \delta_{\mathrm{M}} \ell^{* 2} k, \quad U^{*}=\frac{\epsilon \delta_{\mathrm{R}}^{2} \delta_{\mathrm{M}} \rho^{*} g^{*} \ell^{* 2}}{\mu^{*}} U, \\
V^{*}=\frac{\epsilon \delta_{\mathrm{R}}^{3} \delta_{\mathrm{M}} \rho^{*} g^{*} \ell^{* 2}}{\mu^{*}} V, \quad W^{*}=\frac{\epsilon \delta_{\mathrm{R}}^{3} \rho^{*} g^{*} \ell^{* 2}}{\mu^{*}} W, \quad P^{*}=p_{\mathrm{a}}^{*}+\delta_{\mathrm{R}} \rho^{*} g^{*} \ell^{*} P,
\end{gathered}
$$

where $g^{*}$ denotes the magnitude of acceleration due to gravity and $\ell^{*}=\left[\sigma^{*} /\left(\rho^{*} g^{*}\right)\right]^{1 / 2}$ is the capillary length. Note that $z^{*}$ (but not $x^{*}$ and $y^{*}$ ) is scaled differently in the rivulet and in the membrane. Also note that while the assumption that the rivulet is thin requires that the unscaled contact angle $\beta^{*} \ll 1$ must be small, the scaled contact angle $\beta(>0)$ can take any positive value.

Furthermore, we can choose

$$
\delta_{\mathrm{R}}=\bar{\beta}^{*}, \quad \delta_{\mathrm{M}}=\frac{H^{*}}{\ell^{*}}, \quad \text { and } \quad \epsilon=\frac{k^{*}}{\delta_{\mathrm{R}}^{2} \delta_{\mathrm{M}} \ell^{* 2}}=\frac{k^{*}}{\bar{\beta}^{* 2} \ell^{*} H^{*}},
$$

so that, without loss of generality, $\bar{\beta}=1, H=1$, and $k=1$, although, for clarity of presentation, we retain $\bar{\beta}, H$, and $k$ in the analytical expressions that follow.

Since the membrane is thin, $\delta_{\mathrm{M}} \ll 1$, the leading-order pressure within it satisfies $\partial^{2} P / \partial Z^{2}=0$ subject to $P=p$ at $Z=0$ and $P=0$ at $Z=-H$, with solution

$$
P=p(x, y, 0)\left(1+\frac{Z}{H}\right) \text {. }
$$

Thus, from Darcy's law, the leading-order component of the Darcy velocity in the membrane is solely in the $z$ direction and is given by $\boldsymbol{U}=(0,0, W)$, where

$$
W=-K p(x, y, 0)
$$

where $K$ is a nondimensional membrane permeability defined by $K=k / H$. In particular, this means that the condition of continuity of flux through the base of the rivulet is simply $w=W=-K p$ evaluated on $z=0$, i.e., the local flux through the membrane is proportional to the local value of the pressure at the base of the rivulet.

Since the rivulet is slowly varying, $\epsilon \ll 1$, and thin, $\delta_{\mathrm{R}} \ll 1$, and provided that the appropriately defined reduced Reynolds number $\epsilon \delta_{\mathrm{R}}^{4} \rho^{* 2} g^{*} \ell^{* 3} / \mu^{* 2} \ll 1$ is sufficiently small, the leading-order pressure and velocity within it satisfy the lubrication equations, namely

$$
\frac{\partial u}{\partial x}+\frac{\partial v}{\partial y}+\frac{\partial w}{\partial z}=0, \quad \frac{\partial^{2} u}{\partial z^{2}}+\sin \alpha=0, \quad-\frac{\partial p}{\partial y}=0, \quad-\frac{\partial p}{\partial z}-\cos \alpha=0,
$$

subject to the boundary conditions

$$
u=v=0, \quad w= \begin{cases}0 & \text { for } \quad x \leqslant 0 \\ -K p & \text { for } \quad x>0\end{cases}
$$


on the top surface of the membrane $z=0$,

$$
p=-\frac{\partial^{2} h}{\partial y^{2}}, \quad \frac{\partial u}{\partial z}=\frac{\partial v}{\partial z}=0,
$$

and the kinematic condition

$$
u \frac{\partial h}{\partial x}+v \frac{\partial h}{\partial y}-w=0
$$

on the free surface of the rivulet $z=h$, together with the contact-line conditions

$$
h=0, \quad \frac{\partial h}{\partial y}=\mp \beta
$$

at the contact lines $y= \pm a, z=0$. The solutions of (6) subject to (8) for $u$ and $p$ are

$$
u=\frac{\sin \alpha}{2}\left(2 h z-z^{2}\right), \quad p=(h-z) \cos \alpha-\frac{\partial^{2} h}{\partial y^{2}} .
$$

Since $\partial p / \partial y=0, h$ satisfies

$$
\frac{\partial}{\partial y}\left(\frac{\partial^{2} h}{\partial y^{2}}-h \cos \alpha\right)=0,
$$

subject to (10), which has solution

$$
h=\beta \times \begin{cases}\frac{\cosh m a-\cosh m y}{m \sinh m a} & \text { for } \quad 0 \leqslant \alpha<\frac{\pi}{2} \\ \frac{a^{2}-y^{2}}{2 a} & \text { for } \quad \alpha=\frac{\pi}{2} \\ \frac{\cos m y-\cos m a}{m \sin m a} & \text { for } \quad \frac{\pi}{2}<\alpha \leqslant \pi,\end{cases}
$$

where $m=\sqrt{|\cos \alpha|}$.

The volume flux along the rivulet (i.e., in the positive $x$ direction) is given by

$$
Q=\int_{0}^{h} \int_{-a}^{+a} u d y d z=\frac{\sin \alpha}{3} \int_{-a}^{+a} h^{3} d y=\frac{\beta^{3} \sin \alpha}{9 m^{4}} f(m a),
$$

where the function $f=f(m a)$ originally introduced by Duffy and Moffatt [42] is defined by

$$
f(m a)= \begin{cases}15 m a \operatorname{coth}^{3} m a-15 \operatorname{coth}^{2} m a-9 m a \operatorname{coth} m a+4 & \text { for } 0 \leqslant \alpha<\frac{\pi}{2} \\ \frac{12}{35}(m a)^{4} & \text { for } \alpha=\frac{\pi}{2} \\ -15 m a \cot ^{3} m a+15 \cot ^{2} m a-9 m a \cot m a+4 & \text { for } \frac{\pi}{2}<\alpha \leqslant \pi .\end{cases}
$$

For this solution to be physically realizable we require that $h \geqslant 0$ for $|y| \leqslant a$, and so while there are sessile and vertical rivulets for all values of $a>0$, pendant rivulets occur only for $0<a \leqslant \pi / m$ (see, e.g., Wilson and Duffy [43]).

The thickness of the middle of the rivulet, which from (13) is also the maximum thickness of the rivulet, is denoted by $\bar{h}_{\mathrm{m}}=h(0)$ in $x \leqslant 0$ and $h_{\mathrm{m}}(x)=h(x, 0)$ in $x>0$.

\section{Global conservation of mass}

Global conservation of mass requires that at all stations $x>0$ the volume flux along the rivulet is equal to the sum of the volume flux along the rivulet at $x=0$, namely $\bar{Q}$, and the (negative) volume flux through the base of the rivulet between $x=0$ and the station $x$, i.e.,

$$
Q=\bar{Q}+\int_{0}^{x} \int_{-a(\tilde{x})}^{+a(\tilde{x})} w(\tilde{x}, y, 0) d y d \tilde{x}
$$


Substituting the solution for $p$ given in (11) into the condition of continuity of flux through the base of the rivulet (7) yields

$$
w(x, y, 0)=-K p(x, y, 0)=-K\left(h \cos \alpha-\frac{\partial^{2} h}{\partial y^{2}}\right)=-\frac{K \beta g(m a)}{a} \quad \text { for } \quad x>0,
$$

where the function $g=g(m a)$ is defined by

$$
g(m a)= \begin{cases}m a \operatorname{coth} m a & \text { for } 0 \leqslant \alpha<\frac{\pi}{2} \\ 1 & \text { for } \quad \alpha=\frac{\pi}{2} \\ m a \cot m a & \text { for } \frac{\pi}{2}<\alpha \leqslant \pi,\end{cases}
$$

which is independent of $y$, and so the transverse integral of the normal velocity through the base of the rivulet at the station $x>0$ is

$$
\int_{-a(x)}^{+a(x)} w(x, y, 0) d y=-2 K \beta g(m a) .
$$

Hence the global conservation of mass condition (16) can be written as

$$
\frac{\beta^{3} \sin \alpha}{9 m^{4}} f(m a)=\bar{Q}-2 K \int_{0}^{x} \beta g(m a) d \tilde{x} .
$$

For future reference, it is useful to note that the function $g$ satisfies $g=1+\operatorname{sgn}(\cos \alpha)(m a)^{2} / 3+$ $O(m a)^{4}$ as $m a \rightarrow 0^{+}$, is a monotonically increasing function of $m a$ that satisfies $g \sim m a \rightarrow \infty$ as $m a \rightarrow \infty$ for $0 \leqslant \alpha<\pi / 2$, but is a monotonically decreasing function of $m a$ in the range $0 \leqslant$ $m a \leqslant \pi$ which satisfies $g \sim(\pi / 2)(\pi / 2-m a) \rightarrow 0$ as $m a \rightarrow \pi / 2$ and $g \sim-\pi(\pi-m a)^{-1} \rightarrow-\infty$ as $m a \rightarrow \pi^{-}$for $\pi / 2<\alpha \leqslant \pi$.

Differentiating (20) with respect to $x$ leads to a key result, namely the first-order ordinary differential equation satisfied by $a$ and $\beta$ in $0<x \leqslant L$, i.e.,

$$
\frac{d}{d x}\left[\beta^{3} f(m a)\right]+\frac{18 K m^{4}}{\sin \alpha} \beta g(m a)=0 .
$$

The "nose" (i.e., the downstream end) of the rivulet, located at $x=L$, corresponds to the value of $x$ at which $Q=0$ (i.e., the value of $x$ at which $a=0$ and/or $\beta=0$ ), and hence from (20) the length of the rivulet $L$ is determined by the equation

$$
2 K \int_{0}^{L} \beta g(m a) d x=\bar{Q} .
$$

Note that from (14) evaluated at any station $x \leqslant 0$ the value of $\bar{Q}$ is related to the values of $\bar{a}$ and $\bar{\beta}$ according to

$$
\bar{Q}=\frac{\bar{\beta}^{3} \sin \alpha}{9 m^{4}} f(m \bar{a})
$$

\section{Sessile, vertical, and pendant rivulets}

For sessile and vertical rivulets (i.e., for $0 \leqslant \alpha \leqslant \pi / 2$ ) the pressure at the base of the rivulet, $\beta g(m a) / a$, is always positive, which always drives fluid out of the base of the rivulet and through the membrane. However, for a pendant rivulet (i.e., for $\pi / 2<\alpha \leqslant \pi$ ) this is the case only when $a<a_{\text {crit }}$, where $a_{\text {crit }}=\pi /(2 m)$. In the special case of a pendant rivulet with $a=a_{\text {crit }}$ the pressure at the base of the rivulet is identically zero, and hence no fluid flows either out of or into the rivulet, and so the rivulet is infinitely long and remains uniform with constant semiwidth $a=\bar{a}$ and constant contact angle $\beta=\bar{\beta}$ at all stations $x>0$. For a pendant rivulet with $a>a_{\text {crit }}$ the pressure at the base of the rivulet is negative, which would draw fluid through the membrane and into the base of the rivulet. However, since, as previously noted, in the present work we assume that there is no fluid on the bottom surface of the membrane, this is impossible and so there is no physically realizable rivulet solution in this case. Hence, since the rivulet shrinks as it flows over the permeable part of the 
membrane (and, in particular, since $a \leqslant \bar{a}$ at all stations $x>0$ ), there are sessile and vertical rivulet solutions for all values of $\bar{a}$, whereas there is a pendant rivulet solution only for $\bar{a}<a_{\text {crit }}$ [44]. In particular, for a narrow rivulet whose initial semiwidth satisfies $\bar{a}<\pi / 2$ there is a rivulet solution for all values of $\alpha$, whereas for a wide rivulet whose initial semiwidth satisfies $\bar{a} \geqslant \pi / 2$ there is a rivulet solution only for $0 \leqslant \alpha \leqslant \alpha_{\text {crit }}\left(\pi / 2<\alpha_{\text {crit }} \leqslant \pi\right)$, where

$$
\alpha_{\text {crit }}=\cos ^{-1}\left(-\frac{\pi^{2}}{4 \bar{a}^{2}}\right) \text {. }
$$

\section{E. Area of the base and volume of the rivulet}

In certain contexts, such as some heat-transfer applications, the area of the base of the rivulet on the permeable part of the membrane, denoted by $A$ and scaled and nondimensionalized with $\ell^{* 2} / \epsilon$, and/or the volume of the rivulet on the permeable part of the membrane, denoted by $V$ and scaled and nondimensionalized with $\delta_{\mathrm{R}} \ell^{* 3} / \epsilon$, are of interest, and these are given by

$$
A=2 \int_{0}^{L} a d x
$$

and

$$
V=\int_{0}^{L} \int_{-a}^{+a} \int_{0}^{h} d z d y d x=\frac{2}{\cos \alpha} \int_{0}^{L} \beta[g(m a)-1] d x,
$$

respectively.

\section{A RIVULET WITH FIXED SEMIWIDTH $a \equiv \bar{a}$}

In this section we consider the case of a rivulet with fixed semiwidth $a \equiv \bar{a}$ but decreasing contact angle $\beta=\beta(x)(\leqslant \bar{\beta})$ in $0<x \leqslant L$.

\section{A. Governing equation and solution}

Substituting $a=\bar{a}$ into (21) yields a separable ordinary differential equation for $\beta$, namely

$$
\frac{d\left(\beta^{2}\right)}{d x}=-\frac{12 K m^{4} g(m \bar{a})}{\sin \alpha f(m \bar{a})},
$$

which can be solved subject to the conditions that $\beta(0)=\bar{\beta}$ and $\beta(L)=0$ to yield the explicit solution for $\beta$ and an explicit expression for $L$,

$$
\beta=\bar{\beta}\left(1-\frac{x}{L}\right)^{1 / 2}, \quad L=\frac{\bar{\beta}^{2} \sin \alpha f(m \bar{a})}{12 K m^{4} g(m \bar{a})} .
$$

In the special case of a vertical rivulet (28) reduces to simply

$$
\beta=\bar{\beta}\left(1-\frac{x}{L}\right)^{1 / 2}, \quad L=\frac{\bar{\beta}^{2} \bar{a}^{4}}{35 K} .
$$

Now that $\beta(x)$ and $L$ have been determined, the three-dimensional shape of the free surface of the rivulet, $z=h(x, y)$, is given explicitly by (13).

\section{B. Shape of the rivulet}

Figure 2 shows examples of $\beta$ given by (28) plotted as a function of $x$, and Fig. 3 shows an example of the three-dimensional shape of the free surface of a vertical rivulet. In particular, Fig. 3 illustrates that the rivulet has a "square" nose at which the slope of the free surface of the rivulet in the $x$ direction becomes large according to $d h_{\mathrm{m}} / d x=O(L-x)^{-1 / 2} \rightarrow-\infty$ as $x \rightarrow L^{-}$, and hence that the assumption that the rivulet is slowly varying in $x$ breaks down locally in the vicinity of the nose. 


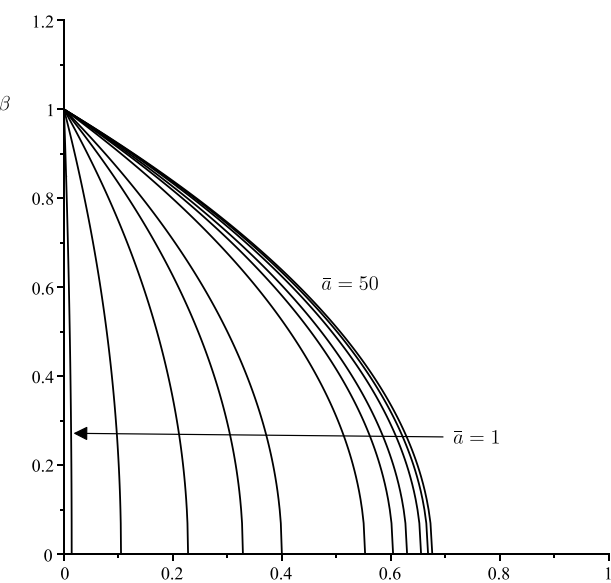

(a)

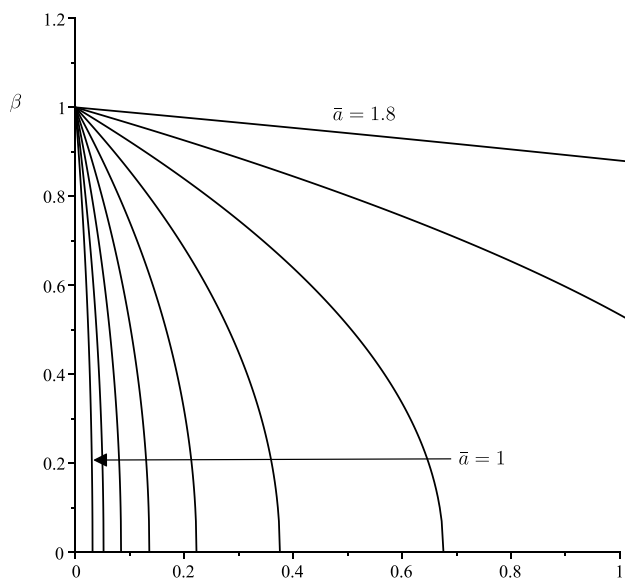

(b)

FIG. 2. The contact angle $\beta$ of a rivulet with fixed semiwidth $a \equiv \bar{a}$ given by (28) plotted as a function of $x$ for (a) a sessile rivulet with $\alpha=\pi / 4$ for $\bar{a}=1,2,3,4,5,10,15,20,30,40,50$, and (b) a pendant rivulet with $\alpha=3 \pi / 4$ for $\bar{a}=1,1.1,1.2, \ldots, 1.8\left(<a_{\text {crit }} \simeq 1.8680\right)$.

\section{Variation of $L$ with $\bar{a}$}

Figure 4 shows examples of $L$ plotted as a function of $\bar{a}$ for a range of values of $\alpha$, including (a) sessile and (b) vertical and pendant rivulets. Figure 4 shows that in all three cases the rivulet becomes short according to

$$
L \sim \frac{\bar{\beta}^{2} \sin \alpha}{35 K} \bar{a}^{4} \rightarrow 0^{+}
$$

in the limit of a very narrow rivulet $\bar{a} \rightarrow 0^{+}$and that the length of the rivulet always increases with $\bar{a}$, but that the behavior for larger values of $\bar{a}$ is qualitatively different for sessile, vertical, and pendant rivulets. Specifically, while Fig. 4(a) shows that the length of a sessile rivulet increases towards an asymptotic value which is reached from below according to

$$
L \sim \frac{\bar{\beta}^{2} \sin \alpha}{2 K m^{4}}\left(1-\frac{11}{6 m \bar{a}}\right) \rightarrow\left(\frac{\bar{\beta}^{2} \sin \alpha}{2 K m^{4}}\right)^{-}
$$

in the limit of a wide rivulet $\bar{a} \rightarrow \infty$, Fig. 4(b) shows that vertical and pendant rivulets become infinitely long according to

$$
L \sim \frac{\bar{\beta}^{2}}{35 K} \bar{a}^{4} \rightarrow \infty \quad \text { as } \quad \bar{a} \rightarrow \infty
$$

and

$$
L \sim \frac{2 \bar{\beta}^{2} \sin \alpha}{3 K \pi m^{5}}\left(a_{\text {crit }}-\bar{a}\right)^{-1} \rightarrow \infty \quad \text { as } \quad \bar{a} \rightarrow a_{\text {crit }}^{-},
$$

respectively. Note that, since $\bar{Q}$ given by (23) is a monotonically increasing function of $\bar{a}$, Fig. 4 may also be interpreted as showing the variation of $L$ with $\bar{Q}$.

In particular, Eq. (31) provides the expression for the length of a two-dimensional (i.e., an infinitely wide) sessile sheet flowing over and through a permeable membrane, namely

$$
L=\frac{\bar{\beta}^{2} \sin \alpha}{2 K m^{4}}=\frac{\bar{h}_{\mathrm{m}}^{2} \tan \alpha}{2 K},
$$

where $\bar{h}_{\mathrm{m}}=\bar{\beta} / m$ is the initial thickness of the sheet, which is in agreement with the corresponding expression given by Davis and Hocking [12] provided that their erroneous scaling of the membrane 


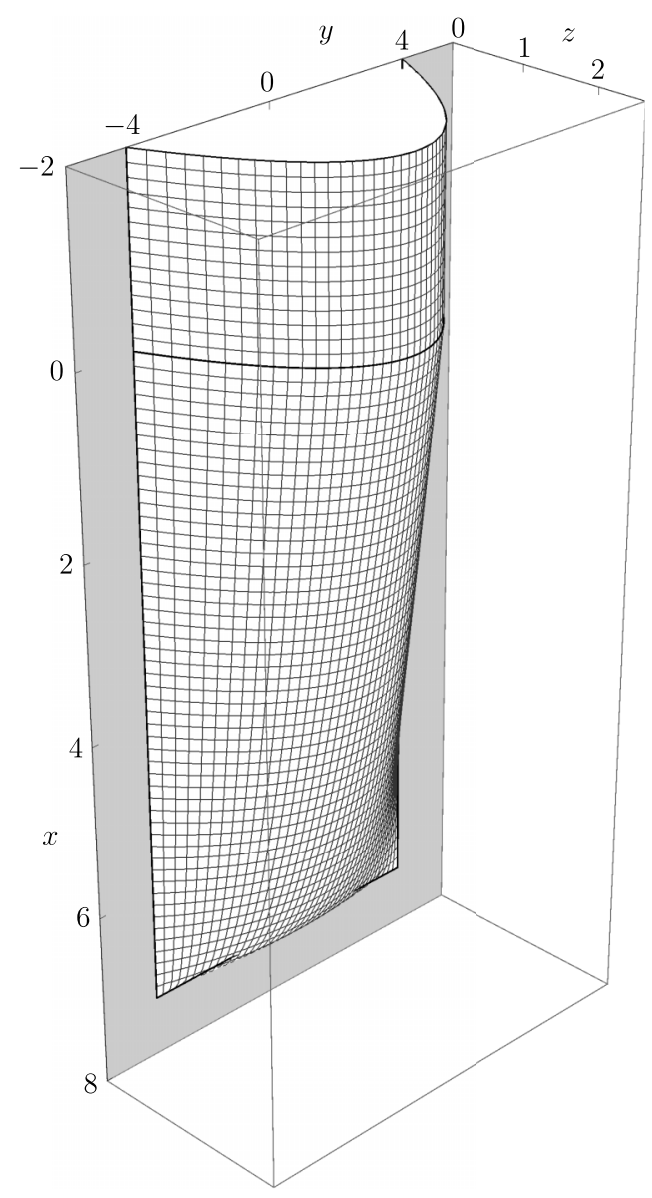

FIG. 3. The three-dimensional shape of the free surface of a vertical rivulet with fixed semiwidth $a \equiv \bar{a}=4$, $\bar{\beta}=1$, and length $L=\bar{\beta}^{2} \bar{a}^{4} /(35 K)=256 / 35 \simeq 7.3143$.

permeability is corrected [46]. Note that the pressure within a two-dimensional vertical sheet is identically zero, and so such a vertical sheet is infinitely long with uniform thickness, while the pressure within a two-dimensional pendant sheet is negative, and so there is no physically realizable pendant sheet solution.

\section{Variation of $L$ with $\alpha$}

Figure 5 shows examples of $L$ plotted as a function of $\alpha / \pi$ for a range of values of $\bar{a}$, including both (a) narrow (i.e., $\bar{a}<\pi / 2$ ) and (b) wide (i.e., $\bar{a} \geqslant \pi / 2$ ) rivulets. Figure 5 shows that in both cases the rivulet becomes short according to

$$
L \sim \frac{\bar{\beta}^{2} f(\bar{a})}{12 K g(\bar{a})} \alpha \rightarrow 0^{+}
$$

in the limit of a sessile rivulet on a nearly horizontal membrane $\alpha \rightarrow 0^{+}$, but that the behavior for larger values of $\alpha$ is qualitatively different for narrow and wide rivulets. Specifically, while Fig. 5(a) shows that the length of a narrow rivulet increases to a global maximum value $L=L_{\max }$ at 


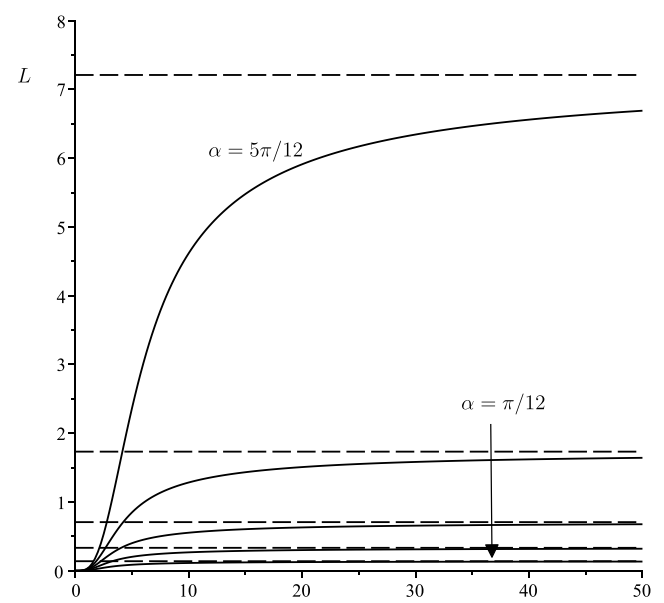

(a)

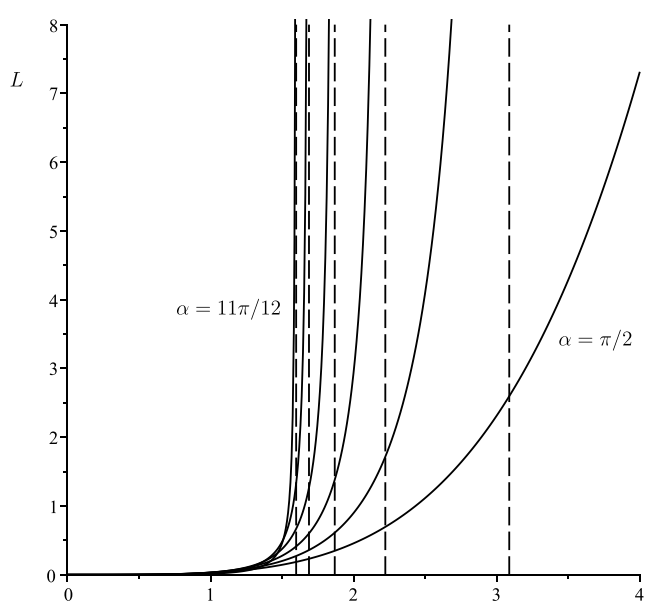

(b)

FIG. 4. The length $L$ of a rivulet with fixed semiwidth $a \equiv \bar{a}$ given by (28) plotted as a function of $\bar{a}$ for (a) sessile rivulets with $\alpha=\pi / 12, \pi / 6, \pi / 4, \pi / 3,5 \pi / 12(<\pi / 2)$ and (b) vertical and pendant rivulets with $\alpha=\pi / 2,7 \pi / 12,2 \pi / 3,3 \pi / 4,5 \pi / 6,11 \pi / 12(\geqslant \pi / 2)$. The dashed lines denote the asymptotic values of $L$ in the limit $\bar{a} \rightarrow \infty$ for sessile rivulets in part (a), and the vertical asymptotes of $L$ at $\bar{a}=a_{\text {crit }}$ for pendant rivulets in part (b).

$\alpha=\alpha_{\max }\left(\pi / 2<\alpha_{\max }<\pi\right)$ and then becomes short again according to

$$
L \sim \frac{\bar{\beta}^{2} f(\bar{a})}{12 K g(\bar{a})}(\pi-\alpha) \rightarrow 0^{+}
$$

in the limit of a pendant rivulet on a nearly horizontal membrane $\alpha \rightarrow \pi^{-}$, Fig. 5(b) shows that the length of a wide rivulet always increases with $\alpha$, and wide rivulets become infinitely long according

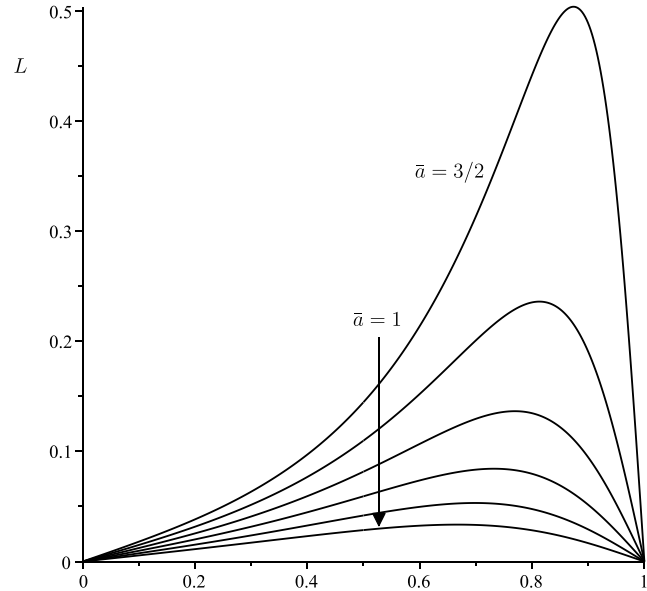

(a)

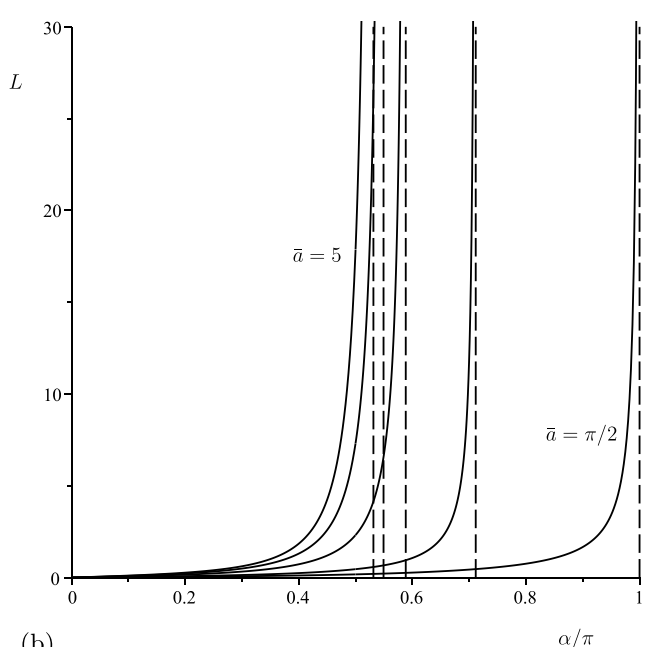

(b)

FIG. 5. The length $L$ of a rivulet with fixed semiwidth $a \equiv \bar{a}$ given by (28) plotted as a function of $\alpha / \pi$ for (a) narrow rivulets with $\bar{a}=1,1.1,1.2,1.3,1.4,1.5(<\pi / 2)$ and (b) wide rivulets with $\bar{a}=\pi / 2,2,3,4,5$ $(\geqslant \pi / 2)$. The dashed lines in part (b) denote the vertical asymptotes of $L$ at $\alpha=\alpha_{\text {crit }}$. 


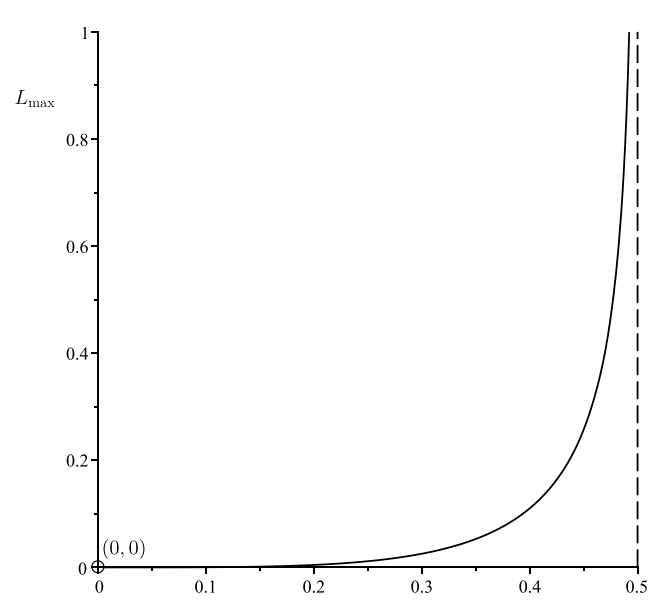

(a)

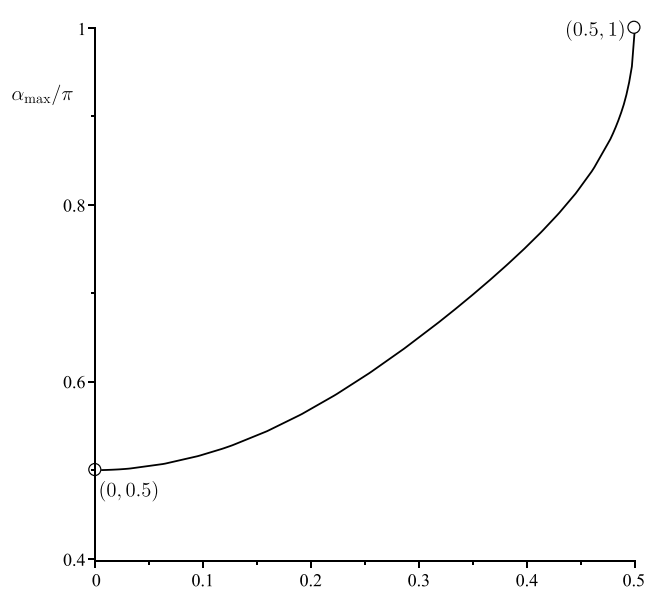

(b)

FIG. 6. (a) $L_{\max }$ and (b) $\alpha_{\max } / \pi$ for a narrow rivulet with fixed semiwidth $a \equiv \bar{a}$ plotted as functions of $\bar{a} / \pi$. The dashed line in part (a) denotes the vertical asymptote of $L_{\max }$ at $\bar{a}=\pi / 2$.

to

$$
L \sim \frac{32 \bar{\beta}^{2} \bar{a}^{2}}{3 K \pi^{4}}\left(\alpha_{\text {crit }}-\alpha\right)^{-1} \rightarrow \infty \quad \text { as } \quad \alpha \rightarrow \alpha_{\text {crit }}^{-}
$$

Figure 6 shows (a) $L_{\max }$ and (b) $\alpha_{\max } / \pi$ for a narrow rivulet plotted as functions of $\bar{a} / \pi$, and, in particular, confirms that $L_{\max }$ and $\alpha_{\max }$ are monotonically increasing functions of $\bar{a}$, and shows that $L_{\max }$ and $\alpha_{\max }$ increase from $L_{\max } \rightarrow 0^{+}$and $\alpha_{\max }=(\pi / 2)^{+}$in the limit $\bar{a} \rightarrow 0^{+}$to $L_{\max } \rightarrow \infty$ and $\alpha_{\max } \rightarrow \pi^{-}$in the limit $\bar{a} \rightarrow(\pi / 2)^{-}$.

\section{E. $A$ and $V$}

Substituting $a=\bar{a}$ into the general expressions for the area of the base of the rivulet on the permeable part of the membrane, $A$, given by (25) and the volume of the rivulet on the permeable part of the membrane, $V$, given by (26) yields

$$
A=\frac{\bar{\beta}^{2} \bar{a} \sin \alpha f(m \bar{a})}{6 K m^{4} g(m \bar{a})}, \quad V=\frac{\bar{\beta}^{3} \tan \alpha f(m \bar{a})[g(m \bar{a})-1]}{9 K m^{4} g(m \bar{a})},
$$

respectively. In the special case of a vertical rivulet (38) reduces to

$$
A=\frac{2 \bar{\beta}^{2} \bar{a}^{5}}{35 K}, \quad V=\frac{4 \bar{\beta}^{3} \bar{a}^{6}}{315 K} \text {. }
$$

\section{A RIVULET WITH FIXED CONTACT ANGLE $\beta \equiv \overline{\boldsymbol{\beta}}$}

In this section we consider the case of a rivulet with fixed contact angle $\beta \equiv \bar{\beta}$ but decreasing semiwidth $a=a(x)(\leqslant \bar{a})$ in $0<x \leqslant L$.

\section{A. Governing equation and solution}

Substituting $\beta=\bar{\beta}$ into (21) yields a separable ordinary differential equation for $a$, namely

$$
\frac{d f(m a)}{d x}=-\frac{18 K m^{4}}{\bar{\beta}^{2} \sin \alpha} g(m a)
$$




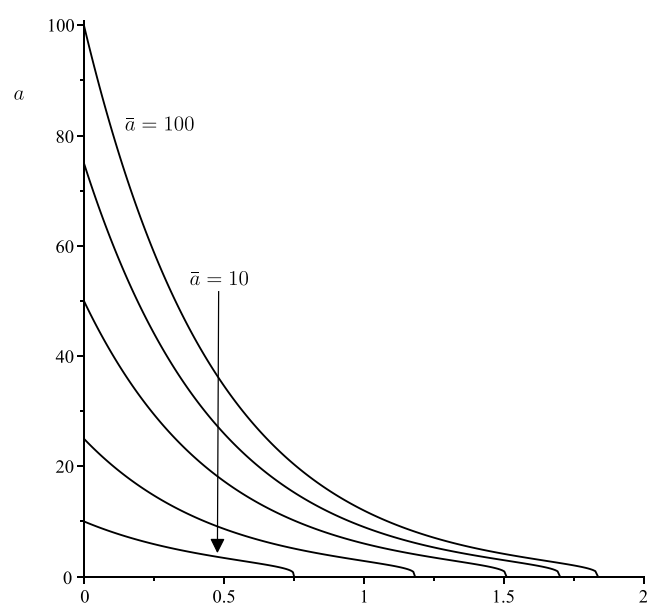

(a)

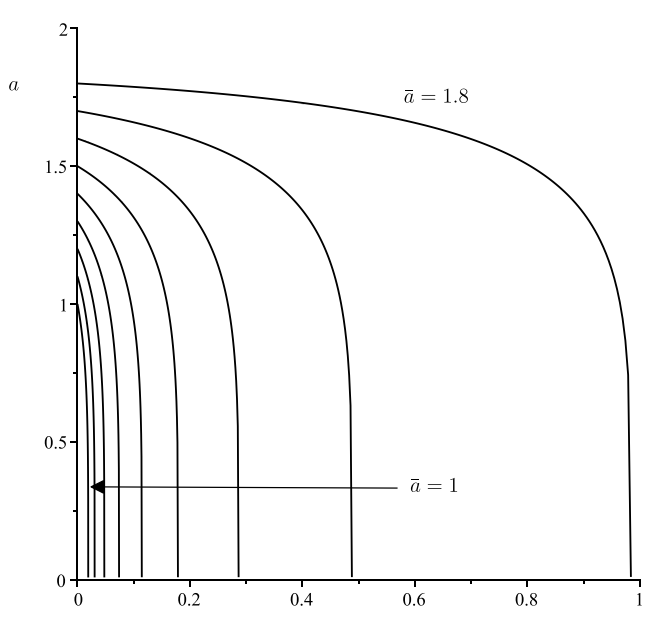

(b)

FIG. 7. The semiwidth $a$ of a rivulet with fixed contact angle $\beta \equiv \bar{\beta}$ given by (41) plotted as a function of $x$ for (a) a sessile rivulet with $\alpha=\pi / 4$ for $\bar{a}=10,25,50,75,100$ and (b) a pendant rivulet with $\alpha=3 \pi / 4$ for $\bar{a}=1,1.1,1.2, \ldots, 1.8\left(<a_{\text {crit }} \simeq 1.8680\right)$.

which can be solved subject to the conditions that $a(0)=\bar{a}$ and $a(L)=0$ to yield the implicit solution for $a$ and an explicit expression for $L$,

$$
F(m a)=\frac{18 K m^{4}}{\bar{\beta}^{2} \sin \alpha}(L-x), \quad L=\frac{\bar{\beta}^{2} \sin \alpha}{18 K m^{4}} F(m \bar{a}),
$$

where the function $F=F(m a)$ is defined by

$$
F(m a)=\int_{0}^{m a} \frac{f^{\prime}(\xi)}{g(\xi)} d \xi
$$

We have not been able to express the function $F(m a)$ in a useful closed form, but it is a function of only one variable which may readily be evaluated by performing the quadrature in (42) numerically. However, it can be shown that $F(m a)$ is a monotonically increasing function of $m a$ which satisfies $F(m a) \sim 12(m a)^{4} / 35 \rightarrow 0^{+}$as $m a \rightarrow 0^{+}, F(m a) \sim 6 \ln (m a) \rightarrow \infty$ as $m a \rightarrow \infty$ for $0 \leqslant \alpha<\pi / 2$, and $F(m a) \sim-9 \ln (\pi / 2-m a) \rightarrow \infty$ as $m a \rightarrow(\pi / 2)^{-}$for $\pi / 2<\alpha \leqslant \pi$. The solution for $a$ can be obtained either by evaluating $F(m a)$ and then solving numerically for $a$ or, equivalently, simply solving the differential equation (40) numerically. However, note that determining the length of the rivulet $L$ given by (41) involves only evaluating $F(m \bar{a})$, and so, slightly surprisingly, does not require us to obtain the solution for $a$. In the special case of a vertical rivulet (41) reduces to an explicit expression for $a$ and the corresponding explicit expression for $L$,

$$
a=\bar{a}\left(1-\frac{x}{L}\right)^{1 / 4}, \quad L=\frac{2 \bar{\beta}^{2} \bar{a}^{4}}{105 K} .
$$

Note that the length of a vertical rivulet with fixed contact angle given by (43) is exactly $2 / 3$ of the length of a vertical rivulet with fixed semiwidth given by (29).

Once $a(x)$ and $L$ have been determined, the three-dimensional shape of the free surface of the rivulet, $z=h(x, y)$, is given explicitly by (13).

\section{B. Shape of the rivulet}

Figure 7 shows examples of $a$ given by (41) plotted as a function of $x$, and Fig. 8 shows an example of the three-dimensional shape of the free surface of a vertical rivulet. In particular, Fig. 8 


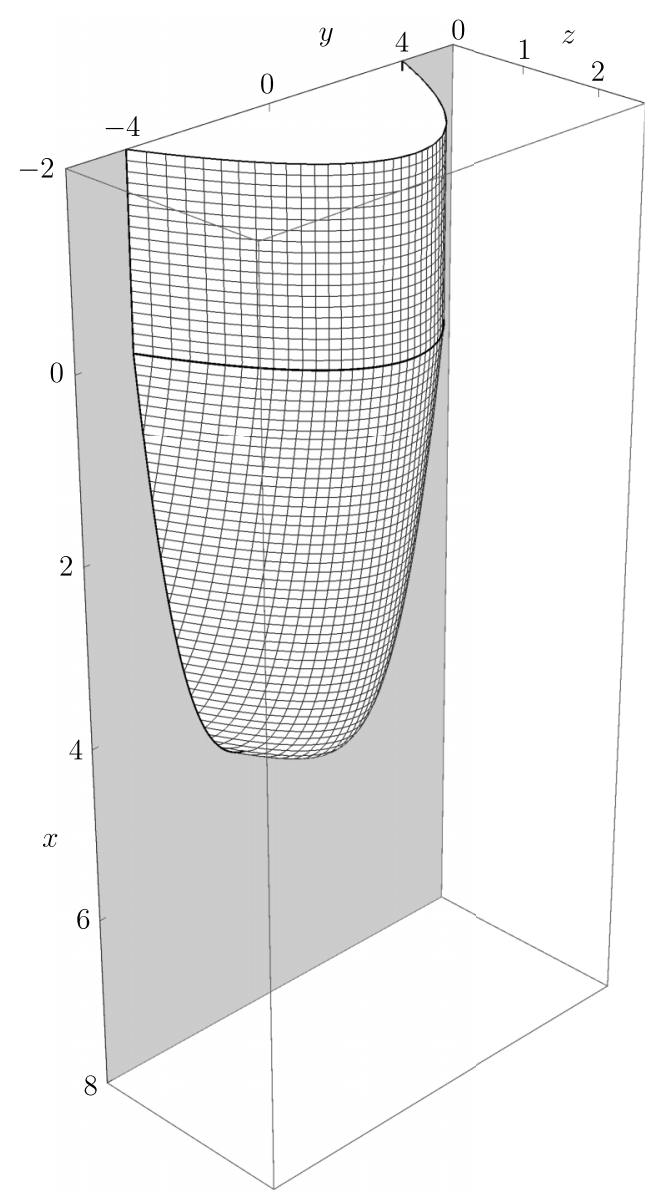

FIG. 8. The three-dimensional shape of the free surface of a vertical rivulet with fixed contact angle $\beta \equiv \bar{\beta}=1, \bar{a}=4$, and length $L=2 \bar{\beta}^{2} \bar{a}^{4} /(105 K)=512 / 105 \simeq 4.8762$.

illustrates that the rivulet has a "rounded" nose given by

$$
a \sim\left[\frac{105 K}{2 \bar{\beta}^{2} \sin \alpha}(L-x)\right]^{1 / 4} \rightarrow 0^{+} \quad \text { as } \quad x \rightarrow L^{-}
$$

at which the slope of the free surface of the rivulet in the $x$ direction becomes large according to $d h_{\mathrm{m}} / d x=O(L-x)^{-3 / 4} \rightarrow-\infty$ as $x \rightarrow L^{-}$, and hence, as for a rivulet with fixed semiwidth, that the assumption that the rivulet is slowly varying in $x$ breaks down locally in the vicinity of the nose.

\section{Variation of $L$ with $\bar{a}$}

Figure 9 shows examples of $L$ plotted as a function of $\bar{a}$ for a range of values of $\alpha$, including (a) sessile and (b) vertical and pendant rivulets. Figure 9 shows that in all three cases the rivulet becomes short according to

$$
L \sim \frac{2 \bar{\beta}^{2} \sin \alpha}{105 K} \bar{a}^{4} \rightarrow 0^{+}
$$




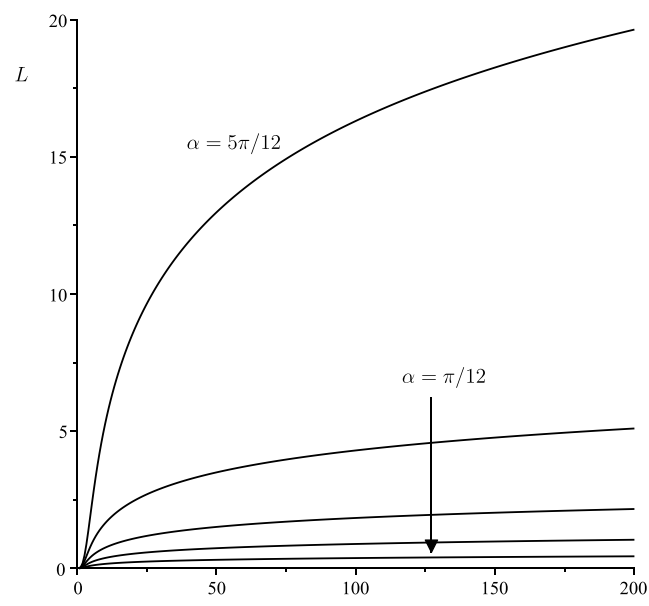

(a)

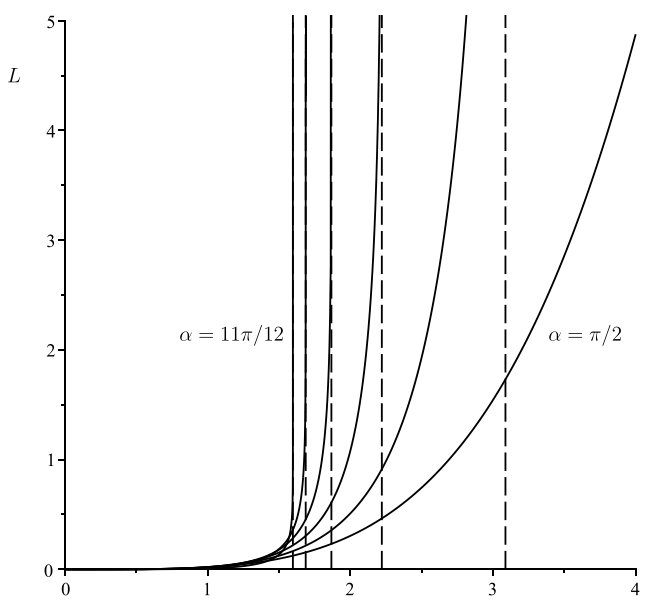

(b)

FIG. 9. The length $L$ of a rivulet with fixed contact angle $\beta \equiv \bar{\beta}$ given by (41) plotted as a function of $\bar{a}$ for (a) sessile rivulets with $\alpha=\pi / 12, \pi / 6, \pi / 4, \pi / 3,5 \pi / 12(<\pi / 2)$ and (b) vertical and pendant rivulets with $\alpha=\pi / 2,7 \pi / 12,2 \pi / 3,3 \pi / 4,5 \pi / 6,11 \pi / 12(\geqslant \pi / 2)$. The dashed lines in part (b) denote the vertical asymptotes of $L$ at $\bar{a}=a_{\text {crit }}$ for pendant rivulets.

in the limit of a very narrow rivulet $\bar{a} \rightarrow 0^{+}$, that the length of the rivulet always increases with $\bar{a}$, and that sessile, vertical, and pendant rivulets all become infinitely long according to

$$
\begin{gathered}
L \sim \frac{\bar{\beta}^{2} \sin \alpha}{3 K m^{4}} \ln (\bar{a}) \rightarrow \infty \quad \text { as } \quad \bar{a} \rightarrow \infty, \\
L=\frac{2 \bar{\beta}^{2}}{105 K} \bar{a}^{4} \rightarrow \infty \quad \text { as } \quad \bar{a} \rightarrow \infty
\end{gathered}
$$

and

$$
L \sim-\frac{\bar{\beta}^{2} \sin \alpha}{2 K m^{4}} \ln \left(a_{\text {crit }}-\bar{a}\right) \rightarrow \infty \quad \text { as } \quad \bar{a} \rightarrow a_{\text {crit }}^{-},
$$

respectively. Note that, like Fig. 4, Fig. 9 may also be interpreted as showing the variation of $L$ with $\bar{Q}$.

\section{Variation of $L$ with $\alpha$}

Figure 10 shows examples of $L$ plotted as a function of $\alpha / \pi$ for a range of values of $\bar{a}$, including both (a) narrow (i.e., $\bar{a}<\pi / 2$ ) and (b) wide (i.e., $\bar{a} \geqslant \pi / 2$ ) rivulets. Figure 10 shows that in both cases the rivulet becomes short according to

$$
L \sim \frac{\bar{\beta}^{2} F(\bar{a})}{18 K} \alpha \rightarrow 0^{+}
$$

in the limit of a sessile rivulet on a nearly horizontal membrane $\alpha \rightarrow 0^{+}$, but that the behavior for larger values of $\alpha$ depends on the value of $\bar{a}$. Specifically, while Fig. 10(a) shows that the length of a narrow rivulet increases to a global maximum value $L=L_{\max }$ at $\alpha=\alpha_{\max }\left(\pi / 2<\alpha_{\max }<\pi\right)$ and then becomes short again according to

$$
L \sim \frac{\bar{\beta}^{2} F(\bar{a})}{18 K}(\pi-\alpha) \rightarrow 0^{+}
$$




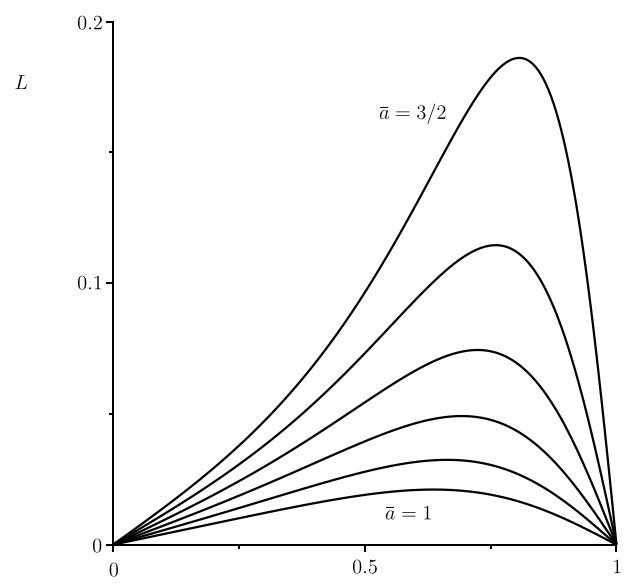

(a)

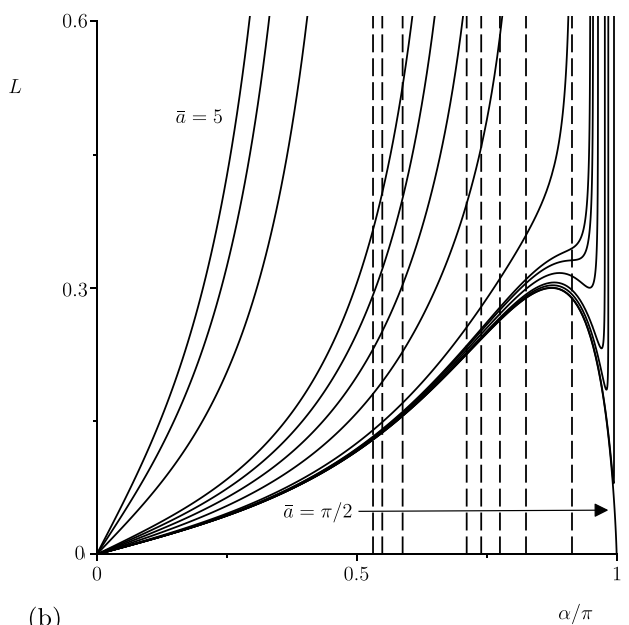

(b)

FIG. 10. The length $L$ of a rivulet with fixed contact angle $\beta \equiv \bar{\beta}$ given by (41) plotted as a function of $\alpha / \pi$ for (a) narrow rivulets with $\bar{a}=1,1.1,1.2,1.3,1.4,1.5(<\pi / 2)$ and (b) wide rivulets with $\bar{a}=\pi / 2,1.571,1.572,1.573,1.576,1.579,1.58,1.6,1.7,1.8,1.9,2,3,4,5(\geqslant \pi / 2)$. The dashed lines in part (b) denote the vertical asymptotes of $L$ at $\alpha=\alpha_{\text {crit }}$ for $\bar{a}=1.6, \ldots, 5$ (the vertical asymptotes for $\bar{a}=\pi / 2, \ldots, 1.58$ having been omitted for clarity).

in the limit of a pendant rivulet on a nearly horizontal membrane $\alpha \rightarrow \pi^{-}$, Fig. 10(b) shows that a wide rivulet becomes infinitely long according to

$$
L \sim-\frac{8 \bar{\beta}^{2} \bar{a}^{2}}{K \pi^{4}} \sqrt{\bar{a}^{4}-\left(\frac{\pi}{2}\right)^{4}} \ln \left(\alpha_{\text {crit }}-\alpha\right) \rightarrow \infty \quad \text { as } \quad \alpha \rightarrow \alpha_{\text {crit }}^{-} .
$$

Figure 11 shows (a) $L_{\max }$ and (b) $\alpha_{\max } / \pi$ for a narrow rivulet plotted as functions of $\bar{a} / \pi$, and, in particular, confirms that $L_{\max }$ and $\alpha_{\max }$ are monotonically increasing functions of $\bar{a}$, and

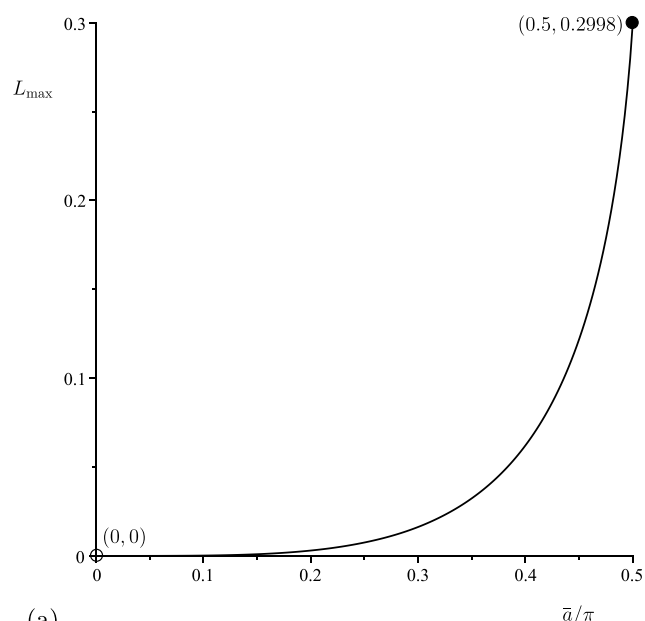

(a)

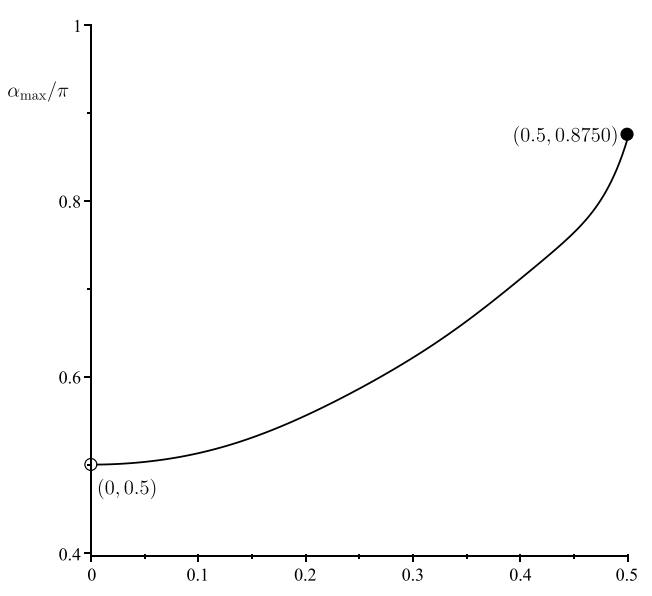

(b)

FIG. 11. (a) $L_{\max }$ and (b) $\alpha_{\max } / \pi$ for a narrow rivulet with fixed contact angle $\beta \equiv \bar{\beta}$ plotted as functions of $\bar{a} / \pi$. 

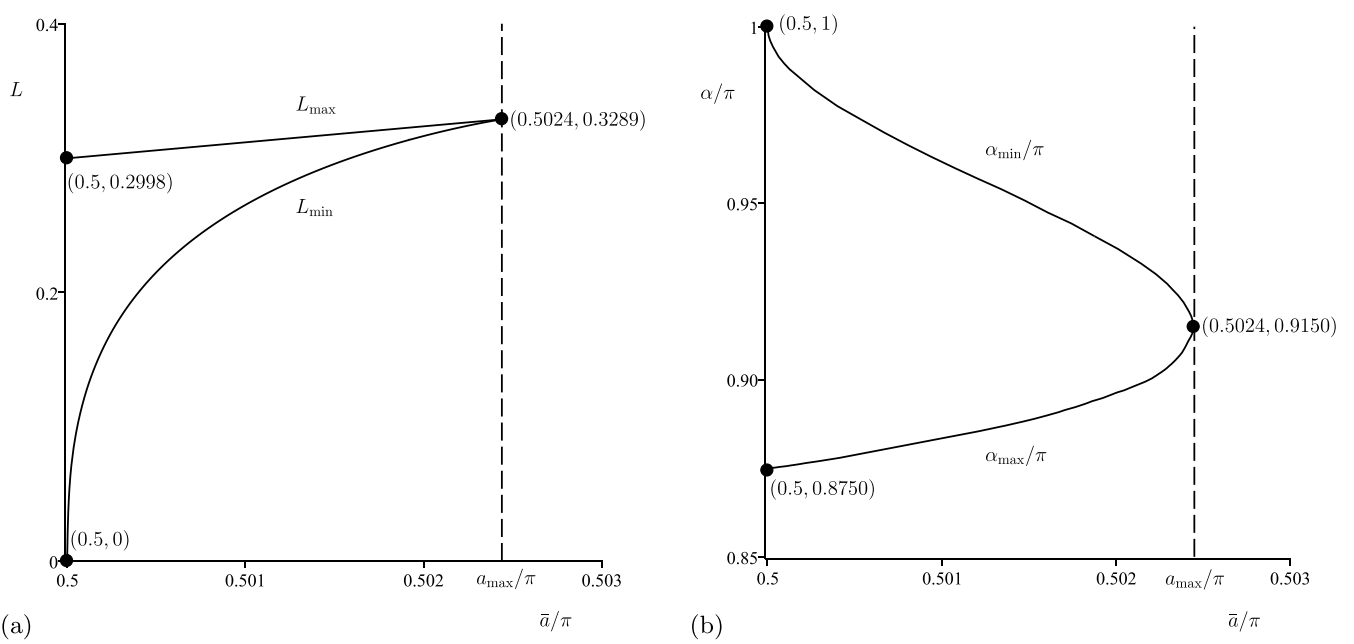

FIG. 12. (a) $L_{\max }$ and $L_{\min }$ and (b) $\alpha_{\max } / \pi$ and $\alpha_{\min } / \pi$ for a wide rivulet with fixed contact angle $\beta \equiv \bar{\beta}$ plotted as functions of $\bar{a} / \pi$.

shows that $L_{\max }$ and $\alpha_{\max }$ increase from $L_{\max } \rightarrow 0^{+}$and $\alpha_{\max } \rightarrow(\pi / 2)^{+}$in the limit $\bar{a} \rightarrow 0^{+}$to $L_{\max }=0.2998$ and $\alpha_{\max } \simeq 0.8750 \pi \simeq 2.7488$ when $\bar{a}=\pi / 2$. However, unlike for a wide rivulet with fixed semiwidth as shown in Fig. 5, Fig. 10(b) also shows that the qualitative behavior of the length of a wide rivulet with fixed contact angle depends on the value of $\bar{a}$. Specifically, as Fig. 10(b) shows, in the narrow range $\pi / 2<\bar{a}<a_{\max }$, where $a_{\max } \simeq 0.5024 \pi \simeq 1.5785$, the length of the rivulet increases to a local maximum value $L=L_{\max }$ at $\alpha=\alpha_{\max }$ and then decreases to a local minimum value $L=L_{\min }$ at $\alpha=\alpha_{\min }\left(\pi / 2<\alpha_{\max }<\alpha_{\min }<\alpha_{\text {crit }}\right)$ before increasing again and becoming infinitely long according to (51) as $\alpha \rightarrow \alpha_{\text {crit }}^{-}$. As Fig. 10(b) also shows, the local maximum and minimum coalesce when $\bar{a}=a_{\max }$, and for $\bar{a} \geqslant a_{\max }$ the length of the rivulet always increases with $\alpha$. Figure 12 shows (a) $L_{\max }$ and $L_{\min }$ and (b) $\alpha_{\max } / \pi$ and $\alpha_{\min } / \pi$ for a wide rivulet plotted as functions of $\bar{a} / \pi$, and, in particular, confirms that $L_{\max }, L_{\min }$, and $\alpha_{\max }$ are monotonically increasing functions of $\bar{a}$ and that $\alpha_{\min }$ is a monotonically decreasing function of $\bar{a}$, and shows that $L_{\max }=L_{\min } \simeq 0.3289, \alpha_{\max }=\alpha_{\min } \simeq 0.9150 \pi \simeq 2.8746$ when $\bar{a}=a_{\max }$.

\section{E. $A$ and $V$}

Substituting $\beta=\bar{\beta}$ into the general expressions for $A$ given by (25) and $V$ given by (26) yields

$$
A=\frac{\bar{\beta}^{2} \sin \alpha}{9 K m^{5}} G(m \bar{a}), \quad V=\frac{\bar{\beta}^{3} \tan \alpha}{9 K m^{4}}[f(m \bar{a})-F(m \bar{a})],
$$

respectively, where the function $G=G(m a)$ is defined by

$$
G(m a)=\int_{0}^{m a} \frac{\xi f^{\prime}(\xi)}{g(\xi)} d \xi
$$

As for the function $F(m a)$, we have not been able to express the function $G(m a)$ in a useful closed form, but, like $F(m a)$, it is a function of only one variable which may readily be evaluated by performing the quadrature in (53) numerically. However, it can be shown that $G(m a)$ is a monotonically increasing function of $m a$ which satisfies $G(m a) \sim 48(m a)^{5} / 175 \rightarrow 0^{+}$as $m a \rightarrow 0^{+}$, $G(m a) \sim 6 m a \rightarrow \infty$ as $m a \rightarrow \infty$ for $0 \leqslant \alpha<\pi / 2$, and $G(m a) \sim-(9 \pi / 2) \ln (\pi / 2-m a) \rightarrow \infty$ 
as $m a \rightarrow(\pi / 2)^{-}$for $\pi / 2<\alpha \leqslant \pi$. In the special case of a vertical rivulet (52) reduces to

$$
A=\frac{16 \bar{\beta}^{2} \bar{a}^{5}}{525 K}, \quad V=\frac{8 \bar{\beta}^{3} \bar{a}^{6}}{945 K} .
$$

Note that the values of $A$ and $V$ for a vertical rivulet with fixed contact angle given by (54) are exactly $8 / 15$ and $2 / 3$, respectively, of the corresponding values for a vertical rivulet with fixed semiwidth given by (39).

\section{CONCLUSIONS}

Motivated by small-scale natural and industrial processes involving flow over and/or through a layer of a porous medium, in the present work we formulated and analyzed a mathematical model for the steady gravity-driven flow of a slowly varying and thin rivulet of fluid with finite width over and through an even thinner permeable membrane.

Expressed in terms of dimensional quantities, the excess pressure (i.e., the pressure above atmospheric pressure) within a rivulet which drives the flow of fluid out of the base of the rivulet and through the membrane is due to a combination of gravitational and capillary effects and is given by

$$
p^{*}-p_{\mathrm{a}}^{*}=\frac{\sigma^{*} \beta^{*}}{a^{*}} g\left(\frac{m a^{*}}{\ell^{*}}\right),
$$

where the function $g(\cdot)$ is defined by (18). This excess pressure is always positive for sessile and vertical rivulets (and hence there are physically realizable sessile and vertical rivulet solutions) for all values of $a^{*}$, but it is positive for a pendant rivulet (and hence there is a physically realizable pendant rivulet solution) only for $a^{*} / \ell^{*}<a_{\text {crit }}=\pi /(2 m)$.

The three-dimensional shape of the free surface of a rivulet with either fixed semiwidth or fixed contact angle was determined, and we showed how the length, base area, and volume of the rivulet on the permeable part of the membrane depend on the physical properties of the system. In particular, we found that the length of a rivulet with fixed semiwidth $a^{*} \equiv \bar{a}^{*}$ is given by

$$
L^{*}=\frac{\sigma^{*} \bar{\beta}^{* 2} H^{*} \sin \alpha}{12 \rho^{*} g^{*} k^{*} \cos ^{2} \alpha} \frac{f\left(m \bar{a}^{*} / \ell^{*}\right)}{g\left(m \bar{a}^{*} / \ell^{*}\right)},
$$

where the function $f(\cdot)$ is defined by (15), and the length of a rivulet with fixed contact angle $\beta^{*} \equiv \bar{\beta}^{*}$ is given by

$$
L^{*}=\frac{\sigma^{*} \bar{\beta}^{* 2} H^{*} \sin \alpha}{18 \rho^{*} g^{*} k^{*} \cos ^{2} \alpha} F\left(\frac{m \bar{a}^{*}}{\ell^{*}}\right),
$$

where the function $F(\cdot)$ is defined by (42). In both cases $L^{*}$ always increases with $\bar{a}^{*}$ and (trivially) with $\bar{\beta}^{*}$. The variation of $L^{*}$ with $\bar{a}^{*}$ is relatively straightforward. A sessile rivulet with fixed semiwidth has a finite maximum possible length (corresponding to the length of a two-dimensional sheet), namely

$$
L^{*}=\frac{\bar{h}_{\mathrm{m}}^{* 2} H^{*} \tan \alpha}{2 k^{*}},
$$

where $\bar{h}_{\mathrm{m}}^{*}\left(=\ell^{*} \bar{\beta}^{*} / m\right)$ is the initial thickness of the sheet, which is attained in the limit of a wide rivulet $\bar{a}^{*} \rightarrow \infty$, but in all of the other situations (i.e., vertical and pendant rivulets with fixed semiwidth and rivulets with fixed contact angle) the length of the rivulet is unbounded in this limit. The variation of $L^{*}$ with $\alpha$ is slightly more complicated. For both a rivulet with fixed semiwidth and a rivulet with fixed contact angle, the length of a narrow rivulet with $\bar{a}^{*} / \ell^{*}<\pi / 2$ increases from zero in the limit of a sessile rivulet on a nearly horizontal membrane $\alpha \rightarrow 0^{+}$to a global maximum value and then decreases back to zero in the limit of a pendant rivulet on a nearly 
horizontal membrane $\alpha \rightarrow \pi^{-}$. On the other hand, the length of a wide rivulet with $\bar{a}^{*} / \ell^{*} \geqslant \pi / 2$ increases from zero in the limit of a sessile rivulet on a nearly horizontal membrane $\alpha \rightarrow 0^{+}$and is unbounded in the limit $\alpha \rightarrow \alpha_{\text {crit }}^{-}$, and, except for a rivulet with fixed contact angle in the narrow range $\pi / 2<\bar{a}^{*} / \ell^{*}<a_{\max } \simeq 0.5024 \pi \simeq 1.5785$, always increases with $\alpha$.

There are a number of ways in which it would be interesting to extend the results described in the present work; notably to consider the situation in which some or all of the fluid adheres to the bottom surface of the membrane, more general boundary conditions on the top surface of the permeable part of the membrane, a thicker membrane within which gravitational effects will play a more significant role, and/or situations in which the contact line of the rivulet on the permeable part of the membrane pins, depins and repins, or, more generally, in which the semiwidth and the contact angle on the permeable part of the membrane vary simultaneously.

\section{ACKNOWLEDGMENTS}

The authors wish to thank Dr. Michael Grinfeld (University of Strathclyde) for his help and support during the course of the present work. A.S.A. wishes to thank the Ministry of Education, Kingdom of Saudi Arabia and King Abdulaziz University for their financial support of his Ph.D. research at the University of Strathclyde in Glasgow.

[1] M. Ungarish and H. E. Huppert, High-Reynolds-number gravity currents over a porous boundary: Shallow-water solutions and box-model approximations, J. Fluid Mech. 418, 1 (2000).

[2] J. M. Acton, H. E. Huppert, and M. G. Worster, Two-dimensional viscous gravity currents flowing over a deep porous medium, J. Fluid Mech. 440, 359 (2001).

[3] D. Pritchard, A. W. Woods, and A. J. Hogg, On the slow draining of a gravity current moving through a layered permeable medium, J. Fluid Mech. 444, 23 (2001).

[4] R. Sayag and J. A. Neufeld, Propagation of viscous currents on a porous substrate with finite capillary entry pressure, J. Fluid Mech. 801, 65 (2016).

[5] Y. Liu, Z. Zheng, and H. A. Stone, The influence of capillary effects on the drainage of a viscous gravity current into a deep porous medium, J. Fluid Mech. 817, 514 (2017).

[6] Y. E. Yu, Z. Zheng, and H. A. Stone, Flow of a gravity current in a porous medium accounting for drainage from a permeable substrate and an edge, Phys. Rev. Fluids 2, 074101 (2017).

[7] E. C. Tredenick, T. W. Farrell, W. A. Forster, and S. T. P. Psaltis, Nonlinear porous diffusion modeling of hydrophilic ionic agrochemicals in astomatous plant cuticle aqueous pores: A mechanistic approach, Front. Plant Sci. 8, 746 (2017).

[8] A. Clarke, T. D. Blake, K. Carruthers, and A. Woodward, Spreading and imbibition of liquid droplets on porous surfaces, Langmuir 18, 2980 (2002).

[9] R. Shamey and X. Zhao, Modelling, Simulation and Control of the Dyeing Process, Woodhead Publishing Series in Textiles (Woodhead Publishing, Cambridge, England, 2014).

[10] K.-C. Park, S. S. Chhatre, S. Srinivasan, R. E. Cohen, and G. H. McKinley, Optimal design of permeable fiber network structures for fog harvesting, Langmuir 29, 13269 (2013).

[11] G. S. White, C. J. W. Breward, P. D. Howell, and R. J. S. Young, A model for the screen-printing of Newtonian fluids, J. Eng. Math. 54, 49 (2006).

[12] S. H. Davis and L. M. Hocking, Spreading and imbibition of viscous liquid on a porous base, Phys. Fluids 11, 48 (1999).

[13] S. H. Davis and L. M. Hocking, Spreading and imbibition of viscous liquid on a porous base. II, Phys. Fluids 12, 1646 (2000).

[14] V. M. Starov, S. R. Kostvintsev, V. D. Sobolev, M. G. Velarde, and S. A. Zhdanov, Spreading of liquid drops over saturated porous layers, J. Colloid Interface Sci. 246, 372 (2002). 
[15] V. M. Starov, S. R. Kostvintsev, V. D. Sobolev, M. G. Velarde, and S. A. Zhdanov, Spreading of liquid drops over dry porous layers: Complete wetting case, J. Colloid Interface Sci. 252, 397 (2002).

[16] N. Allerborn and H. Raszillier, Spreading and sorption of a droplet on a porous substrate, Chem. Eng. Sci. 59, 2071 (2004).

[17] D. M. Anderson, Imbibition of a liquid droplet on a deformable porous substrate, Phys. Fluids 17, 087104 (2005).

[18] K. Nong and D. M. Anderson, Thin film evolution over a thin porous layer: Modelling a tear film on a contact lens, SIAM J. Appl. Math. 70, 2771 (2010).

[19] L. Espín and S. Kumar, Droplet spreading and absorption on rough, permeable substrates, J. Fluid Mech. 784, 465 (2015).

[20] L. Espín and S. Kumar, Droplet wetting transitions on inclined substrates in the presence of external shear and substrate permeability, Phys. Rev. Fluids 2, 014004 (2017).

[21] R. Liu and Q. Liu, Instabilities of a liquid film flowing down an inclined porous plane, Phys. Rev. E 80, 036316 (2009).

[22] H. N. Kandel and J. P. Pascal, Inclined fluid-film flow with bottom filtration, Phys. Rev. E 88, 052405 (2013).

[23] M. Iervolino, J.-P. Pascal, and A. Vacca, Instabilities of a power-law film over an inclined permeable plane: A two-sided model, J. Non-Newtonian Fluid Mech. 259, 111 (2018).

[24] G. Z. Ramon, H. E. Huppert, J. R. Lister, and H. A. Stone, On the hydrodynamic interaction between a particle and a permeable surface, Phys. Fluids 25, 073103 (2013).

[25] D. J. Knox, S. K. Wilson, B. R. Duffy, and S. McKee, Porous squeeze-film flow, IMA J. Appl. Math. 80, 376 (2015).

[26] D. J. Knox, B. R. Duffy, S. McKee, and S. K. Wilson, Squeeze-film flow between a curved impermeable bearing and a flat porous bed, Phys. Fluids 29, 023101 (2017).

[27] D. C. Venerus, Squeeze flows in liquid films bound by porous disks, J. Fluid Mech. 855, 860 (2018).

[28] T. Gambaryan-Roisman, Liquids on porous layers: Wetting, imbibition and transport processes, Curr. Opin. Colloid Interface Sci. 19, 320 (2014).

[29] C. Paterson, S. K. Wilson, and B. R. Duffy, Pinning, de-pinning and re-pinning of a slowly varying rivulet, Eur. J. Mech. B/Fluids 41, 94 (2013).

[30] S. V. Alekseenko, S. P. Aktershev, A. V. Bobylev, S. M. Kharlamov, and D. M. Markovich, Nonlinear forced waves in a vertical rivulet flow, J. Fluid Mech. 770, 350 (2015).

[31] M. A. Herrada, A. S. Mohamed, J. M. Montanero, and A. M. Gañán-Calvo, Stability of a rivulet flowing in a microchannel, Int. J. Multiphase Flow 69, 1 (2015).

[32] P. D. Howell, H. Kim, M. G. Popova, and H. A. Stone, Rivulet flow over a flexible beam, J. Fluid Mech. 796, 285 (2016).

[33] F. H. H. Al Mukahal, B. R. Duffy, and S. K. Wilson, Advection and Taylor-Aris dispersion in rivulet flow, Proc. R. Soc. A 473, 20170524 (2017).

[34] M. Wang, J. Zhao, and R. Duan, Rivulet formulation in the flow of film down a uniformly heated vertical substrate, Eng. Appl. Comput. Fluid Mech. 13, 396 (2019).

[35] A. S. Alshaikhi, S. K. Wilson, and B. R. Duffy, Rivulet flow down a slippery substrate, Phys. Fluids 32, 072011 (2020).

[36] P. G. Ledda, G. Lerisson, G. Balestra, and F. Gallaire, Instability of a thin viscous film flowing under an inclined substrate: The emergence and stability of rivulets, J. Fluid Mech. 904, A23 (2020).

[37] S. Aktershev, S. Alekseenko, and A. Bobylev, Waves in a rivulet falling down an inclined cylinder, AIChE J. 67, e17002 (2021).

[38] Another assumption would be necessary to describe the situation in which some or all of the fluid adheres to the bottom surface of the membrane in the form of droplets and/or a new rivulet. However, in the present work we restrict our attention to the simplest case in which there is no fluid on the bottom surface of the membrane.

[39] Depending on the specific physical situation being modeled, other conditions, such as continuity of velocity in the $x^{*}$ and $y^{*}$ directions, slip conditions (see, e.g., Alshaikhi et al. [35] and the discussion therein), or the Beavers-Joseph condition (see, e.g., the classical work of Beavers and Joseph [40] and 
Saffman [41]), might be more appropriate on the top surface of the permeable part of the membrane. However, in the present work we restrict our attention to the simplest case of no flow in the $x^{*}$ and $y^{*}$ directions on the top surface of both the permeable and the impermeable parts of the membrane.

[40] G. S. Beavers and D. D. Joseph, Boundary conditions at a naturally permeable wall, J. Fluid Mech. 30, 197 (1967).

[41] P. G. Saffman, On the boundary condition at the surface of a porous medium, Stud. Appl. Math. 50, 93 (1971).

[42] B. R. Duffy and H. K. Moffatt, Flow of a viscous trickle on a slowly varying incline, Chem. Eng. J. 60, 141 (1995).

[43] S. K. Wilson and B. R. Duffy, On the gravity-driven draining of a rivulet of viscous fluid down a slowly varying substrate with variation transverse to the direction of flow, Phys. Fluids 10, 13 (1998).

[44] In particular, this means that there is no solution corresponding to a rivulet with zero contact angle, $\beta=0$, since such a rivulet is possible only for $\pi / 2<\alpha \leqslant \pi$ and has semiwidth $a=\pi / m=2 a_{\text {crit }}$ regardless of the value of $\bar{Q}$ (see, e.g., Sullivan et al. [45]).

[45] J. M. Sullivan, S. K. Wilson, and B. R. Duffy, A thin rivulet of perfectly wetting fluid subject to a longitudinal surface shear stress, Q. J. Mech. Appl. Math. 61, 25 (2008).

[46] Specifically, in the notation of Davis and Hocking [12], the dimensionally incorrect scaling of their "permeability parameter" (our membrane permeability) given in their Eq. (36), namely $\mu h_{0}(\beta \tan \theta)^{2 / 3} / 3$, should be $h_{0}(\beta \tan \theta)^{2} / 3$. In addition, there is a missing pair of brackets around $\partial h^{\prime} / \partial t^{\prime}+\partial Q^{\prime} / \partial x^{\prime}$ in the first line of their Eq. (34), $\cos \theta$ should be $\sin \theta$ in their definition of their Bond number $B$, and their final expression for their "survival distance" (corresponding to our rivulet length), $a \sim\left(k^{\prime}\right)^{-1} g^{-1 / 3}$, should be $a^{\prime} \sim\left(k^{\prime}\right)^{-1} h_{0}^{2}$. 